\title{
Post-transcriptional modifications to tRNA-a response to the genetic code degeneracy
}

\author{
YA-MING HOU, ${ }^{1}$ HOWARD GAMPER, ${ }^{1}$ and WEI YANG ${ }^{2}$ \\ ${ }^{1}$ Department of Biochemistry and Molecular Biology, Thomas Jefferson University, Philadelphia, Pennsylvania 19107, USA \\ ${ }^{2}$ Department of Chemistry and Biochemistry, Florida State University, Tallahassee, Florida 32306-4390, USA
}

We used to think of the genetic code degeneracy as an obligatory process resulting from assigning 61 sense codons to the 20 amino acids in protein. In this degeneracy, 18 of the 20 amino acids are endowed with multiple codons, and the conventional wisdom was that each of the 18 was translated equally from each codon. However, due to the degeneracy mostly manifest at the wobble position for each amino acid, we were led to believe that the degeneracy is simply to provide a deck of codons for safety control, in case one or two codons are damaged by nucleotide substitutions to the wobble position. However, work over a long path has now shown that the degeneracy has a much deeper meaning, with the ability to confer codon-by-codon translational regulation.

At its core, translation of a codon requires pairing interaction with the anticodon of its cognate tRNA. Due to the degeneracy, multiple codons are paired with one anticodon in different efficiencies and stabilities. This is a form of translational regulation executed at the level of individual codons. Nature has developed this translational regulation by two means: one by employing biased codon usage and the other by modifying the chemical structure of the tRNA anticodon or adjacent nucleotides via post-transcriptional modifications. While biased codon usage is genetically encoded, post-transcriptional modifications are epigenetic and have more flexibility to modulate the fidelity and efficiency of codon-anticodon pairing interactions. Indeed, among codons for the same amino acid, some require tRNA modifications that are stress-induced, conferring a capacity to regulate cellular adaptation to stress at individual codons. Thus, in response to the genetic code degeneracy, tRNA has evolved to carry post-transcriptional modifications to fine-tune translation one codon at a time. Enzymes catalyzing tRNA modifications are often linked to human diseases, ranging from metabolic defects, mitochondrial dysfunctions, and neurological disorders, to cancer.

Corresponding author: ya-ming.hou@jefferson.edu

Article and publication date are at http://www.rnajournal.org/cgi/doi/ 10.1261/rna.049825.115. Freely available online through the RNA Open Access option.
The greatest diversity of tRNA modifications is at positions 34 (the wobble position) and 37 (on the $3^{\prime}$ side of the anticodon), two positions critical for translational accuracy and reading-frame maintenance. Three modifications at these positions have strong effects on codon-anticodon pairing interactions. First, U34 is modified to 5-methoxycarbonyl-methyl-2-thiouridine $\left(\mathrm{mcm}^{5} \mathrm{~s}^{2} \mathrm{U} 34\right)$ in $\mathrm{tRNA}^{\mathrm{Ly} / \mathrm{UUU}}$ (anticodon $5^{\prime}$-UUU-3'), tRNA ${ }^{\text {Glu/UUC, }}$ tRNA ${ }^{\text {Gln/UUG }}$, and tRNA ${ }^{\mathrm{Arg} / \mathrm{UCU}}$. In the case of tRNA ${ }^{\mathrm{Lys} / \mathrm{UUU}}$ for example, while it can read both Lys codons (5'-AAA and 5'-AAG), the codon-anticodon pairing in either case is weak, consisting of A-U and G-U base pairs. The addition of $\mathrm{mcm}^{5} \mathrm{~s}^{2}$ to U34 can stabilize the A-U and G-U pairs at the wobble position by providing an extended chemical group to enhance the stacking interaction within each pair. The importance of this $\mathrm{mcm}^{5} \mathrm{~s}^{2}$ modification is emphasized by its response to cellular stress. Specifically, $\mathrm{mcm}^{5} \mathrm{~s}^{2} \mathrm{U} 34$ is synthesized in two sequential steps, first via the addition of $\mathrm{mcm}^{5}$ to U34 by the ELP complex and second via the addition of $\mathrm{s}^{2}$ to the $\mathrm{mcm}^{5} \mathrm{U} 34$ intermediate by the URM1 pathway. While the ELP complex is formally associated with the RNA pol II transcription complex in yeast, it catalyzes the $\mathrm{mcm}^{5}$ addition to U34 upon cellular oxidative stress. Similarly, the URM1 complex is activated by cellular stress in yeast, such as oxidative stress and temperature up-shift stress. Recent work shows that $\mathrm{mcm}^{5} \mathrm{~s}^{2}$ addition to U34 facilitates translation of codonbiased mRNAs required for stress response. For example, upon stress with the alkylating agent methyl methane sulfonate, the methyl transferase Trm9 associated with the ELP complex is activated to help to translate DNA damage response genes each with a pattern of codon usage dependent on $\mathrm{mcm}^{5} \mathrm{~s}^{2} \mathrm{U} 34$-tRNA for translation. Similarly, a human Trm9-like protein in cancer cells helps to translate the LIN9 protein, which has a codon bias requiring $\mathrm{mcm}^{5} \mathrm{~s}^{2} \mathrm{U} 34-$ tRNA for translation. LIN9 interacts with the tumor suppressor protein $\mathrm{Rb}$ to suppress cancer progression, thus implicating the Trm9-like protein and the $\mathrm{mcm}^{5} \mathrm{~s}^{2}$ modification of U34-tRNA with a role in inhibition of tumor growth.

(c) 2015 Hou et al. This article, published in $R N A$, is available under a Creative Commons License (Attribution-NonCommercial 4.0 International), as described at http://creativecommons.org/licenses/by-nc/4.0/. 
A second example involves 5-methylation to cytosine $\left(\mathrm{m}^{5} \mathrm{C}\right)$ of $\mathrm{tRNA}{ }^{\mathrm{Leu} / \mathrm{CAA}}$. Although $\mathrm{m}^{5} \mathrm{C}$ is present in at least 34 yeast tRNA species, it is at the wobble position only in tRNA $^{\text {Leu/CAA }}$ as well as at position 48 in the $\mathrm{V}$ loop of the same tRNA. Upon cellular oxidative stress, however, the distribution of $\mathrm{m}^{5} \mathrm{C}$ in the tRNA is dynamically changed, with a significant increase at the wobble position and a concomitant decrease at position 48 , indicating a specific response to the stress. In yeast, the methyl transferase Trm4 is responsible for synthesis of $\mathrm{m}^{5} \mathrm{C} 34$ to help decode the UUG codon. Without the modification, the codon-anticodon pairing interaction is weak and is dominated by A-U base pairs, whereas the presence of the modification provides a methyl group to enhance the stacking interaction at the wobble position. In yeast, the importance of $\mathrm{m}^{5} \mathrm{C} 34$ for translation of the UUG codon is correlated with the highly biased distribution of UUG in genes involved in oxidative stress response. One of these genes is for the ribosomal protein Rpl22a, where all Leu residues are coded by UUG. Indeed, yeast cells exposed to oxidative stress preferentially synthesize Rpl22a, rather than its paralog Rpl22b, where only $34 \%$ of Leu residues are coded by UUG. The preferential synthesis of Rpl22a may confer adaptation to oxidative stress by several mechanisms, such as activating translation via the internal ribosomal entry site, regulating $\mathrm{T}$-cell development, or engaging in the activity of the non-ribosomal complex of telomerase.

A third example is based on $\mathrm{N}^{1}$-methylation to G37 $\left(\mathrm{m}^{1} \mathrm{G} 37\right)$ of tRNA ${ }^{\text {Pro/GGG }}$. The cognate codon for this tRNA is CCC and the codon-anticodon pairing interaction is stable, consisting of three G-C base pairs. Nonetheless, without the modification, tRNA ${ }^{\text {Pro/GGG }}$ is highly prone to +1 -frameshifting, particularly on the slippery CC[C/U]-[C/ $\mathrm{U}]$ sequences in mRNA. Taking the CCC-C mRNA sequence for example; the high propensity of the tRNA for making a +1 frameshift $(+1 \mathrm{FS})$ is in part due to its identical codon-anticodon pairing interactions in the un-shifted 0 -frame and in the shifted +1 -frame. Given that $\mathrm{CC}[\mathrm{C} / \mathrm{U}]-[\mathrm{C} / \mathrm{U}]$ sequences occur $\sim 2500$ times among protein-coding genes in E. coli, this poses a serious challenge for reading-frame maintenance. Unlike missense errors, +1FS errors are unforgiving; causing premature termination of protein synthesis and generating truncated products likely toxic to cells. The $\mathrm{m}^{1} \mathrm{G} 37$ modification is known to suppress +1FS errors, although the mechanism has remained unresolved for a long time. Recent data from x-ray crystal structural work and advanced biochemical analysis have begun to shed light on the mechanism of suppression, showing that $\mathrm{m}^{1} \mathrm{G} 37$ induces structural re-arrangement of the tRNA anticodon loop to restrict both the dynamics and kinetics of $+1 \mathrm{FS}$ errors. Unexpectedly, while $\mathrm{m}^{1} \mathrm{G} 37$ is conserved among all three isoacceptors of tRNA $^{\text {Pro }}$ (anticodons GGG, CGG, and UGG), its strength in suppressing +1FS errors varies; it is dominant and sufficient by itself in the UGG isoacceptor but is relatively weak in the GGG isoacceptor and requires the multi-faceted elongation factor EF-P for assistance. In the UGG isoacceptor, the wobble U34 is naturally modified to $\mathrm{cmo}^{5} \mathrm{U} 34$ (uridine-5oxyacetic acid), which enables strong pairing to CCA and CCG but weak pairing to CCC and CCU of Pro codons. The dominance of $\mathrm{m}^{1} \mathrm{G} 37$ in the UGG isoacceptor may have arisen to prevent weak codon-anticodon pairs from +1 -frameshifting.

The examples of $\mathrm{mcm}^{5} \mathrm{~s}^{2} \mathrm{U} 34, \mathrm{~m}^{5} \mathrm{C} 34$, and $\mathrm{m}^{1} \mathrm{G} 37$ illustrate that each post-transcriptional modification to tRNA is a response to the genetic code degeneracy and consequently the multiplicity of tRNAs for specific amino acid. In each case, the key question of how the modification stabilizes an otherwise unstable codon-anticodon pair remains to be addressed. The study of $\mathrm{m}^{1} \mathrm{G} 37$ can provide a framework for moving forward; specifically, the role of $\mathrm{m}^{1} \mathrm{G} 37$ was elucidated from analysis of each tRNA ${ }^{\text {Pro }}$ in three states: (i) the G37state made by in vitro transcription, lacking any post-transcriptional modification, (ii) the $\mathrm{m}^{1} \mathrm{G} 37$-state made by in vitro transcription, followed by an enzyme-catalyzed synthesis of the modified base, and (iii) the native-state isolated from cells, containing all natural post-transcriptional modifications. These three states were compared for individual reaction steps of protein synthesis using purified ribosomes and translation factors. In parallel, crystal structures of $\mathrm{m}^{1} \mathrm{G} 37$ tRNA on the ribosome were solved and genomic occurrences of the cognate $\mathrm{CC}[\mathrm{C} / \mathrm{U}]-[\mathrm{C} / \mathrm{U}]$ mRNAs were determined from bioinformatics. It is by combining data of these diverse approaches that an integrated view of how $\mathrm{m}^{1} \mathrm{G} 37$ works begins to emerge. This example demonstrates the power of using complementary tools and methodologies to elucidate the mechanism and function of each tRNA modification.

Future work should focus more on tRNA modifications in multi-cellular organisms. While several modifications are conserved in all kingdoms of life, most show few phenotypes in single-cell organisms. For example, deficiency of $\mathrm{mcm}^{5} \mathrm{~s}^{2} \mathrm{U} 34$ and $\mathrm{m}^{5} \mathrm{C} 34$ in single-cell organisms has no phenotype in normal growth conditions, but manifests growth defects in cellular stress. The exception is $\mathrm{m}^{1} \mathrm{G} 37$, whose deficiency is sufficient to arrest cell growth of both E. coli and yeast even without external stress. In contrast, deficiency of $\mathrm{mcm}^{5} \mathrm{~s}^{2} \mathrm{U} 34$ and $\mathrm{m}^{5} \mathrm{C} 34$ elicits diverse human diseases, indicating a stronger role of these modifications in human cells. For example, lack of the ELP complex for the first step synthesis of $\mathrm{mcm}^{5} \mathrm{~s}^{2} \mathrm{U} 34$ results in severe human neurological defects, including the familial dysautonomia syndrome (causing patients to lose sensory neurons), intellectual disabilities, and non-familial amyotrophic lateral scelerosis. Similarly, deficiencies of $\mathrm{m}^{5} \mathrm{C} 34$ in $\mathrm{tRNA}^{\mathrm{Leu} / \mathrm{CAA}}$, due to pathogenic mutations in NSUN2 (the homolog of yeast Trm4), are associated with intellectual disabilities and a Dubowitzlike syndrome with mild microcephaly and congenital heart defects. Notably, in addition to these neurological disorders, deficiencies in tRNA modifications are linked to human diseases with metabolic defects, mitochondrial dysfunctions, and cancer. To date, very few human tRNA modifications are characterized; we anticipate that efforts directed 
at elucidating human tRNA modifications at the enzymatic and mechanistic level will pave the way to understand how modifications are associated with human diseases and to gain insights into therapeutic interventions.

\section{Acknowledgments}

This work is supported by NIH R01GM108972 (to Y.-M.H.) and NIH R01GM111886 (to W.Y.). 

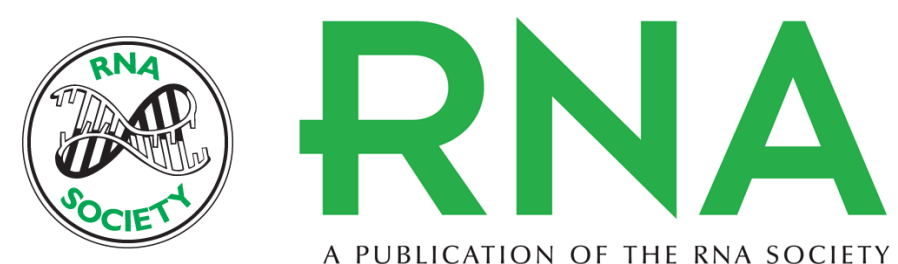

A PUBLICATION OF THE RNA SOCIETY

\section{Post-transcriptional modifications to tRNA--a response to the genetic code degeneracy}

Ya-Ming Hou, Howard Gamper and Wei Yang

RNA 2015 21: 642-644

Open Access Freely available online through the RNA Open Access option.

Creative This article, published in RNA, is available under a Creative Commons License

Commons (Attribution-NonCommercial 4.0 International), as described at

License http://creativecommons.org/licenses/by-nc/4.0/.

Email Alerting Receive free email alerts when new articles cite this article - sign up in the box at the

Service top right corner of the article or click here.

To subscribe to $R N A$ go to:

http://rnajournal.cshlp.org/subscriptions

(C) 2015 Hou et al.; Published by Cold Spring Harbor Laboratory Press for the RNA Society 